\title{
CLASSES OF $p$-VALENT STARLIKE FUNCTIONS
}

\author{
G. R. BLAKLEY 1
}

1. Introduction. The winding number associated with a starlike function exhibits a certain monotonicity property (Theorem 1 below). This property is used to show that several alternatives to the definition of the class $S(p)$ of $p$-valent starlike functions are trivial. From it there also follows a simple and explicit example of a coefficient problem in $S(p)$ with no solution. This situation, which Goodman has treated in some detail [2], is interesting since such problems always have solutions in the schlicht case $[3 ; 4]$.

Let $S$ be the class of all functions $f(z)=z+a_{2} z^{2}+a_{3} z^{3}+\cdots$ which are regular and schlicht in $|z|<1$ and let $S^{*}$ be the subclass of $S$ consisting of those functions whose image domains are starshaped with respect to the origin. For a given positive integer $p$ let $S(p)$, the class of $p$-valent starlike functions, be the class of all functions $f$ to which there corresponds some $r, 0<r<1$, such that for any $z, r<|z|<1$, $\operatorname{Re}\left\{z f^{\prime}(z) / f(z)\right\} \geqq 0$ and $(1 / 2 \pi) \int_{0}^{2 \pi} \operatorname{Re}\left\{z f^{\prime}(z) / f(z)\right\} d t=p, z=q e^{i t}$, for each $q, r<q<1$. This integral is just the number of zeros of $f$ in the interior of the circle $|z|=q$ and hence $f$ has $p$ zeros in the open unit disk, and is in fact $p$-valent there [2]. In a certain sense the classes $S(1)$ and $S^{*}$ coincide, i.e., if $f \in S^{*}$ then $f \in S(1)$ and if $f \in S(1)$ then $f / f^{\prime}(0) \in S^{*}$.

2. The winding number. If $Q$ is a path in $U=\{z:|z|<1\}$ and $f$ is analytic in $U$, let $f(Q)$ denote the path which is the image of $Q$ under $f$ and which has the induced orientation. The properties of the winding number

$$
n[f(Q), a]=\frac{1}{2 \pi i} \oint_{Q} \frac{f^{\prime}(z)}{f(z)-a} d z
$$

are well known. In this paper $f$ will lack singularities and $Q$ will be a circle. Hence $n[f(Q), a]$ will be the number of times $f$ assumes the value $a$ in the open disk bounded by $Q$.

If a function $f$ is regular at a point $a \neq 0$ and $\operatorname{Re}\left\{z f^{\prime}(z) / f(z)\right\} \geqq 0$ for all $z$ in some neighborhood $M$ of $a$ then the fact that $\operatorname{Re}\left\{z f^{\prime}(z) / f(z)\right\}$ is harmonic in some neighborhood $N \subset M$ of $a$ implies that $\operatorname{Re}\left\{a f^{\prime}(a) / f(a)\right\}>0$ and, consequently, also that $f^{\prime}(a) \neq 0$. From this fact, which will be of frequent use below, follows

Received by the editors September 6, 1960 and, in revised form, January 3, 1961.

1 This research was supported by the Office of Ordnance Research, U. S. Army, under Contract No. DA-36-034-ORD-1486 with the University of Maryland. 
THEOREM 1. Let the function $f$ be regular in the open unit disk $U$ and zero at the origin. Suppose there is an $r=r(f), 0<r<1$, such that (i) $f(z) \neq 0$ and (ii) $\operatorname{Re}\left\{z f^{\prime}(z) / f(z)\right\} \geqq 0$, whenever $r<|z|<1$. Then for any $q, r<q<1$, and associated $Q=\{z:|z|=q\}$ and any $a \in U$, the winding number $n[f(Q), s f(a)]$ is a decreasing function of the positive real variable $s$ as long as $s f(a) \in f(U)$.

Proof. Let $f(U)$ be the image of $U$ under $f$. The winding number $n[f(Q), s f(a)]$ is a nonnegative integer (hence real) and is constant throughout each component of $f(U)$ determined by $f(q)$. Therefore, as has just been noted, $\operatorname{Re}\left\{z f^{\prime}(z) / f(z)\right\}=\partial \arg f(z) / \partial \arg z>0$ whenever $r<|z|<1$, i.e. $\arg f$ is a strictly increasing function of $\arg z$ for $z \in Q$. Furthermore the fact that $f$ is never zero in $\{z: r<|z|<1\}$ and has only a finite number $m>0$ of zeros in $\{z:|z| \leqq r\}$ implies, with the help of the argument principle, that arg $f(z)$ increases by $2 m \pi$ as $z$ makes one positively directed circuit of $Q$. Thus $\operatorname{Arg} f(z)$ takes on each value $b, 0 \leqq b<2 \pi$ precisely $m$ times as $z$ traverses $Q$.

If $a \in Q$ is arbitrary it is apparent that the angle $\theta$ from the radius vector $f(a)-0$ to the vector tangent to $f(Q)$ at $f(a)$ lies in the interval $0<\theta<\pi$. The geometric meaning of the winding number now makes it obvious that its value falls as $f(Q)$ is crossed in an outward direction and in fact that this decrease is just some integer $n, 1 \leqq n \leqq m$, which is the number of points of $Q$ mapped into $f(a)$ by $f$. The proof of the theorem is now complete.

3. The class $M_{p}^{*}$.

Definition 1. Let $p$ be fixed, $p \geqq 1$. Suppose the function $h(z)$ $=z^{p}+b_{p+1} z^{p+1}+b_{p+2} z^{p+2}+\cdots$ is regular in $U$ and satisfies the conditions

(i) $h$ is $p$-valent in $U$, and

(ii) there exists $r=r(h), 0<r<1$, such that $\operatorname{Re}\left\{z h^{\prime}(z) / h(z)\right\} \geqq 0$ whenever $r<|z|<1$.

Then $h$ is called a function of class $M_{p}^{*}$.

Manifestly if $h \in M_{p}^{*}$ then for any $q, r<q<1$, and $Q=\{z:|z|=q\}$ it must be that $n[h(Q), 0]=p$, i.e., $(1 / 2 \pi) \int_{0}^{2 \pi} \operatorname{Re}\left\{z h^{\prime}(z) / h(z)\right\} d t=p$, $z=q e^{i t}$, so that $h \in S(p)$. Thus $M_{p}^{*} \subset S(p)$. Let $\left(S^{*}\right)^{p}$ be the class of $p$ th powers of functions of $S^{*}$. Then

Theorem 2. $M_{p}^{*}=\left(S^{*}\right)^{p}, \quad p=1,2,3, \cdots$.

Proof. Choose any function

$$
\begin{aligned}
h(z) & =z^{p}+b_{p+1} z^{p+1}+b_{p+2} z^{p+2}+\cdots \\
& =z^{p}\left(1+b_{p+1} z+b_{p+2} z^{2}+\cdots\right)=z^{p} g(z)
\end{aligned}
$$


of $M_{p}^{*}$. Note that $g$ is regular and nonzero in $U$. Hence so is $[g(z)]^{1 / p}$. Since $g(0)=1$ the function $[g(z)]^{1 / p}$ can be assumed to be 1 at the origin. Set $f(z)=z[g(z)]^{1 / p}=z+\cdots$ Then $[f(z)]^{p}=h(z)$. Furthermore, if $z \in U, z h^{\prime}(z) / h(z)=p\left(z f^{\prime}(z) / f(z)\right)$ whence, since $p>0$, $\operatorname{Re}\left\{z f^{\prime}(z) / f(z)\right\} \geqq 0$ whenever $r(h)=r<|z|<1$. Hence, just as above, $\arg f(z)$ is a strictly increasing function of arg $z$ for $z \in Q$. Therefore $f(Q)$ is a simple closed curve and by Darboux's Theorem $f$ is schlicht. Thus $f \in S^{*}$, and consequently $M_{p}^{*} \subset\left(S^{*}\right)^{p}$.

If, now, $f(z)=z+a_{2} z^{2}+a_{3} z^{3}+\cdots$ is a member of $S^{*}$ and the function $h(z)=z^{p}+\cdots$ is defined by setting $h(z)=[f(z)]^{p}$ then certainly $h \in M_{p}^{*}$, for $f$ can take on each $p$ th root of a given number at most once in $U$, showing that $h$ satisfies condition (i). That $h$ satisfies condition (ii) has already been shown in the first half of this proof. Therefore $\left(S^{*}\right)^{p} \subset M_{p}^{*}$ and the theorem is proved.

\section{The class $N_{p}^{*}$.}

Definition 2. Let $p$ be fixed, $p \geqq 1$. Let $m$ be an integer, $1 \leqq m \leqq p$. Suppose the function $h(z)=z^{m}+b_{m+1} z^{m+1}+b_{m+2} z^{m+2}+\cdots$ is regular in $U$ and satisfies the conditions

(i) $h$ is at most $p$-valent in $U$, and

(ii) $\operatorname{Re}\left\{z h^{\prime}(z) / h(z)\right\} \geqq 0$ for all $z \in U$.

Then $h$ is called a function of class $N_{p}^{*}$.

The relationship of the class $N_{p}^{*}$ to $S(p)$ is made plain by the following

Theorem 3. $N_{p}^{*}=S^{*} \cup\left(S^{*}\right)^{2} \cup \cdots \cup\left(S^{*}\right)^{p}, \quad p=1,2,3, \cdots$.

Proof. Consider an arbitrary $h \in N_{p}^{*}$. The function $h$ is of the form $h(z)=z^{m}+b_{m+1} z^{m+1}+b_{m+2} z^{m+2}+\cdots$ for some integer $m, 1 \leqq m \leqq p$. To show that $h \in M_{m}^{*}=\left(S^{*}\right)^{m}$ it clearly suffices to verify that $h$ is $m$-valent in $U$. Consider any point $a \neq 0$ of $U$. If $h(a)=0$ then, in some neighborhood of $a, h(z)=(z-a)^{n} g(z)$ where $1 \leqq n$ and $g(a) \neq 0$ (for the identically zero function is not a member of $N_{p}^{*}$ ). Then $z h^{\prime}(z) / h(z)=n a /(z-a)+n+z g^{\prime}(z) / g(z)$. Certainly $n+z g^{\prime}(z) / g(z)$ is analytic at $z=a$ since $g(a) \neq 0$ and therefore $z h^{\prime}(z) / h(z)$ has a pole of order 1 at $z=a$ in contradiction to condition (ii) in the hypothesis concerning $h$. Thus $h$ can be zero in $U$ only at the origin. Hence for any $q, 0<q<1$, and associated $Q=\{z:|z|=q\}$ and $G=\{z:|z|<q\}$ it is apparent that $n[h(Q), h(0)]=n[h(Q), 0]=m$. Theorem 1 now guarantees that $n[h(Q), h(z)] \leqq m$ whenever $z \in G$. Since $q$ can be arbitrarily close to $1, h$ must be $m$-valent in $U$. Thus $N_{p}^{*} \subset S^{*}$ $\cup\left(S^{*}\right)^{2} \cup \ldots \cup\left(S^{*}\right)^{p}$.

For $m=1,2,3, \cdots, p$ the proof that $\left(S^{*}\right)^{m} \subset N_{p}^{*}$ is the same as 
the corresponding part of Theorem 2. This establishes the opposite inclusion and therewith the theorem.

5. The class $S_{p}^{*}$.

Definition 3. Let $p=1,2,3, \cdots$. Suppose the function $f(z)$ $=z+a_{2} z^{2}+a_{3} z^{3}+\cdots$ is regular in $U$ and satisfies the conditions

(i) $f$ is at most $p$-valent in $U$,

(ii) there exists some $a \in f(U)$ such that $f(z)=a$ exactly $p$ times in $U$, and

(iii) there exists $r=r(f), 0<r<1$, such that $\operatorname{Re}\left\{z f^{\prime}(z) / f(z)\right\} \geqq 0$ whenever $r<|z|<1$.

Then $f$ is called a function of class $S_{p}^{*}$.

Evidently $S_{1}^{*}=S^{*}$ which, in turn, is in the sense above alluded to just equal to $S(1)$. But not even in this sense is $S_{p}^{*}=S(p)$. However

Theorem 4. $S_{p}^{*} \subset S(p), \quad p=1,2,3, \cdots$.

Proof. If $f \in S_{p}^{*}$ then all that must be verified to show that $f \in S(p)$ is the existence of a $d, 0<d<1$, such that whenever $d<q<1$

$$
n[f(Q), 0]=\frac{1}{2 \pi} \int_{0}^{2 \pi} \operatorname{Re}\left\{\frac{z f^{\prime}(z)}{f(z)}\right\} d t=p,
$$

$z=q e^{i t}$.

There are some $a \in U$, some number $k, 0<k<1$, and a circle $K=\{z:|z|=k\}$ contained in $U$ such that $n[f(K), f(a)]=p$. Let $c, 0<c<1$, be the largest of the moduli of the (at most $p$ ) points $z_{j}$ of $U$ at which $f\left(z_{j}\right)=0$. The number $r=r(f)$ is already associated with $f$. Define $d=\max \{r, k, c\}$. Then for any $q, d<q<1$, and associated $Q=\{z:|z|=q\}$ it is true that $n[f(Q), f(a)]=p$. The fact that $f$ is at most $p$-valent in $U$ implies that $n[f(Q), 0] \leqq p$. Hence an application of Theorem 1 to $f$ yields $p=n[f(Q), f(a)] \leqq n[f(Q), 0] \leqq p$. Since $q$ can be arbitrarily close to 1 this shows that $f \in S(p)$, i.e. $S_{p} \subset S(p)$, which completes the proof.

If $g$ is any function of $S(p)$ having a simple zero at the origin then $g$ can be written $g(z)=a_{1} z+a_{2} z^{2}+a_{3} z^{3}+\cdots$. It is now a trivial matter to verify that

$$
\frac{g(z)}{a_{1}}=\frac{g(z)}{g^{\prime}(0)}=z+\frac{a_{2}}{a_{1}} z^{2}+\frac{a_{3}}{a_{1}} z^{8}+\cdots
$$

is an element of $S_{p}^{*}$. And in this sense, the same as with the classes $S(1)$ and $S^{*}=S_{1}^{*}$ (i.e. except for normalization) the class $S_{p}^{*}$ is just the class of all functions of $S(p)$ having a simple zero at the origin. 
It is clear that for $p=2,3,4, \cdots$ no function of $S_{p}^{*}$ is the power of a (schlicht) starlike function.

The following theorems give an example of a coefficient problem which has no solution.

THEOREM 5. Let $p$ be fixed, $p \geqq 2$. If $k$ is any complex number such that $2-1 / p<|k|$ then the function $f(z)=z+k z^{p}$ is a member of $S_{p}^{*}$.

Proof. The function $f$ has $p$ zeros in $U$ and is obviously $p$-valent in $U$. Also, for $|z|=1$,

$$
\begin{aligned}
\operatorname{Re}\left\{\frac{z f^{\prime}(z)}{f(z)}\right\} & =\operatorname{Re}\left\{p+\frac{(1-p) z}{z+k z^{p}}\right\} \geqq p-\frac{p-1}{|k|-1} \\
& =\frac{p}{k-1}\left(|k|-2+\frac{1}{p}\right)>0
\end{aligned}
$$

which persists, by continuity, for $r<|z|<1$, for some $r, 0<r<1$. Therefore $f \in S_{p}^{*}$.

Theorem 6. Let $p$ be fixed, $p \geqq 2$. Let $n \neq p$ be chosen, $n \geqq 2$. Then to any complex number $q$ there corresponds a function $f(z)=z+a_{2} z^{2}$ $+a_{3} z^{3}+\cdots$ of $S_{p}^{*}$ for which $a_{n}=q$.

Proof. If $q=0$ the theorem is an immediate consequence of Theorem 5. So consider arbitrary fixed nonzero $q$, and let $b$ be any complex number whose modulus satisfies the inequality $1+|q|<p+|q|$ $+|n-p| \cdot|q|<|b|$. Now consider the function $f(z)=z+b z^{p}+q z^{n}$. In consequence of the above inequality and a theorem of Pellet [ 1 , p. 10] on roots of polynomials it follows immediately that $n[f(C), 0]$ $=p$, where $C=\{z:|z|=1\}$. The fact that $\max _{z \in C}\left|z f^{\prime}(z) / f(z)-p\right|$ $<1<p$ implies, just as in the proof of Theorem 5 , that there exists $d$, $0<d<1$, such that $\operatorname{Re}\left\{z f^{\prime}(z) / f(z)\right\} \geqq 0$ whenever $d<|z|<1$. Since $f$ takes on only $p$ zeros in $U$ there exists $t, 0<t<1$, such that $f(z) \neq 0$ whenever $t<|z|<1$. Set $r=\max (d, t)$. Then $0<r<1$ and Theorem 1 is applicable to $f$. Hence if $r<x<1$ and $X=\{z:|z|=x\}$ the winding number $n[f(X), s f(a)]$ is a decreasing function of the positive real variable $s$ whenever $s f(a) \in f(U)$. Thus $p=n[f(C), 0]=n[f(X), 0]$ $\geqq n[f(X), k]$ for any $k \in f(U)$. But $x$ can be arbitrarily close to 1 . Therefore $f \in S_{p}^{*}$ and the theorem is proved.

Consideration of the classes of $p$-valent starlike functions treated above has given rise to the following question concerning a decomposition for elements of $S(p)$. Given any $f \in S(p)$, does $f$ have a representation $f=g h$ where $g \in S_{p}^{*}, h \in\left(S^{*}\right)^{p-1}$ ? 


\section{REFERENCES}

1. M. J. Dieudonne, La theorie analytique des polynomes d'une variable, GauthierVillars, Paris, 1938.

2. A. W. Goodman, On the Schwars-Christoffel transformation and p-valent functions, Trans. Amer. Math. Soc. 68 (1950), 204-233.

3. J. A. Hummel, The coefficient regions of starlike functions, Pacific J. Math. 7 (1957), 1381-1389.

4. - A variational method for starlike functions, Proc. Amer. Math. Soc. 9 (1958), 82-87.

UNIVERSITY OF MARYLAND AND

Cornell University 